\title{
Highly Nondegenerate Two-Photon Absorption in Silicon Wire Waveguides
}

\author{
Nicolas Poulvellarie, ${ }^{1,2}$ Charles Ciret, ${ }^{3}$ Bart Kuyken, ${ }^{2}$ François Leo, ${ }^{1}$ and Simon-Pierre Gorza ${ }^{1, *}$ \\ ${ }^{1}$ OPERA-Photonique, Université libre de Bruxelles, 50 Avenue Franklin D. Roosevelt, CP 194/5, 1050 Brussels, \\ Belgium \\ ${ }^{2}$ Department of Information Technology, Photonics Research Group, Ghent University-IMEC, 9000 Ghent, \\ Belgium \\ ${ }^{3}$ Laboratoire de Photonique d'Angers EA 4464 Université d'Angers, 2 Boulevard Lavoisier, 49000 Angers, France
}

(Received 17 May 2018; published 22 August 2018)

\begin{abstract}
Nondegenerate two-photon absorption (ND TPA) is studied in a nanophotonic silicon waveguide in a configuration such that the dispersion of the nonlinear absorption and refraction cannot be ignored. It is shown that a signal wave around $1330 \mathrm{~nm}$ can be strongly absorbed by ND TPA with a pulse of 6-W peak power at $1850 \mathrm{~nm}$. Experiments are performed with pulses of about $200 \mathrm{fs}$ in a dispersion engineered waveguide in which the pump and signal pulses propagate at a similar group velocity. The experimental results are very well reproduced by numerical simulations of two coupled generalized nonlinear Schrödinger equations (GNLSEs). We show that the nonlinear dynamics can be well described by a single GNLSE despite the wavelength separation between the pump and the signal waves. We also demonstrate that in silicon nanophotonic waveguides, the dispersion of the nonlinear absorption is much larger than the dispersion of the Kerr effect. This could have an impact in the design of all-optical functions based on ND TPA, as well as on the study of supercontinuum and frequency-comb generation in integrated semiconductor-on-insulator platforms.
\end{abstract}

DOI: 10.1103/PhysRevApplied.10.024033

\section{INTRODUCTION}

Silicon nanophotonic waveguides have attracted significant interest owing to their large nonlinear optical response. Nonlinear optical functionalities such as alloptical signal processing [1], wavelength conversion [2,3], and supercontinuum generation [4,5] have been demonstrated. At telecom wavelengths, crystalline silicon, however, suffers from nonlinear absorption, which limits its performance. Nevertheless, the two-photon-absorption (TPA) process in silicon can be exploited for ultrashortpulse characterization [6], ultrafast all-optical switching [7] and modulation [8,9], mode locking [10], or singlecycle pulse generation [11]. Moreover, it has recently been pointed out that nonlinear absorption plays a central role in the soliton-fission mechanism at the heart of supercontinuum generation (SCG) from subpicosecond pulses [12].

Nondegenerate two-photon absorption (ND TPA) is the nonlinear mechanism by which two photons of different waves are simultaneously absorbed in a material. The dispersion properties of the degenerate TPA coefficient in crystalline silicon are well known $[13,14]$ and have been confirmed by many experiments. However, despite the importance of the nondegenerate nonlinear absorption

\footnotetext{
*sgorza@ulb.ac.be
}

process in broadband applications, little is known about its properties when the interacting wavelengths are vastly different. Results in silicon have been reported [15-17]. However, in these studies the two interacting waves were separated by only $75 \mathrm{MHz}, 5 \mathrm{~nm}$, and $30 \mathrm{~nm}$, respectively, and the approximation of a constant TPA coefficient is relevant. In direct-band-gap semiconductors, in contrast to indirect-band-gap semiconductors, ND TPA has been extensively studied both theoretically and experimentally [18]. It is now well known that owing to the sharp absorption-band edge of direct-gap semiconductors, TPA for extreme nondegenerate cases involving spectrally distant waves can be very large. Enhancements by a factor of up to 1000 have been experimentally demonstrated as compared with degenerate TPA. This has been applied for the efficient detection of mid-infrared radiation mediated by a gating pulse $[18,19]$. Such enhancements are not expected in indirect-gap semiconductors because of the slow dependence of the linear absorption coefficient with frequency close to the indirect-band-gap energy [18]. The common approximation is to consider the total energy of the two photons involved to compute the ND TPA (or the cross-phase-modulation) coefficient in indirect-band-gap semiconductors. This means that $\chi^{(3)}\left(-\omega_{s} ; \omega_{p},-\omega_{p}, \omega_{s}\right)$ is replaced by its degenerate value at the average frequency of the two waves; that is, at $\left(\omega_{p}+\omega_{s}\right) / 2$ [20]. Photons of energy close to or below the half-band-gap could thus 
experience a large cross-absorption with photons in the telecom windows. Moreover, since the nonlinear absorption coefficient strongly depends on the frequency, its dispersion properties could have a non-negligible impact on the nonlinear wave propagation, potentially larger than the dispersion of the Kerr effect.

In this work, our aim is to experimentally investigate in indirect-band-gap semiconductor waveguides the ND TPA between spectrally distant pulses. Our experiment thus serves as a test bed for assessing the validity of the simplifying assumption that infers the cross nonlinear coefficients from their degenerate values at the average frequency. We also discuss the ability of the single generalized nonlinear Schrödinger equation to describe the nonlinear interaction between the two pulses, despite their large wavelength separation. Our motivation stems from the fact that this equation is commonly used to model the nonlinear dynamics of broadband waves in integrated nonlinear platforms.

\section{EXPERIMENT}

Integrated semiconductor nanophotonic waveguides are the ideal platform for implementing low-power nonlinear optical functions owing to the high confinement of the light. In addition, the geometry of the waveguide allows the dispersion properties to be engineered. These properties play a central role in ensuring efficient nonlinear interactions. They may also allow two distant wavelengths to have the same group velocity. This is particularly useful for increasing the interaction length between short pulses. The dispersion of the waveguide used in the experiment is shown in Fig. 1 for a quasi-TE mode. The curve shows that waves of around 1850 and $1300 \mathrm{~nm}$ propagate with a similar group velocity, but with anomalous and normal dispersion, respectively. A soliton pulse, excited at around $1850 \mathrm{~nm}$, may hence strongly interact with a signal pulse of around $1300 \mathrm{~nm}$. Optical-event horizons have been studied in a similar configuration [21,22].

The experimental setup is shown in Fig. 2. The two interacting pulses are generated by an optical parametric

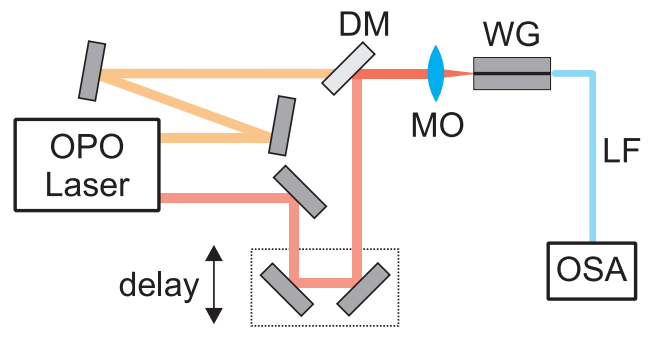

FIG. 2. Experimental setup. OPO: optical parametric oscillator, DM: dichroic mirror, MO: microscope objective, WG: siliconon-insulator nanophotonic waveguide, LF: lensed fiber, OSA: optical spectrum analyzer.

oscillator, synchronously pumped by a mode-locked titanium-sapphire laser at $82 \mathrm{MHz}$. The input pump power at $1848 \mathrm{~nm}$ is close to the power required to excite a fundamental soliton. The weaker pulse of around $1300 \mathrm{~nm}$ acts as a signal wave, and the dominant contribution to its nonlinear absorption comes from the cross-interaction with the pump. Before the coupling into the silicon nanophotonic waveguide, these two beams are combined on a dichroic mirror with a controllable delay. We consider a 2cm-long, 220-nm-high, 780-nm-wide silicon-on-insulator wire waveguide. At the waveguide output, the light is collected by means of a lensed fiber and sent to an optical spectrum analyzer (either a Yokogawa AQ6370 optical spectrum analyzer for measuring the signal spectrum or an OSA203B Fourier transform optical spectrum analyzer from Thorlabs for the pump). The lensed fiber position is set to optimize the amplitude of the collected signal wave. The input pulse profiles are characterized by means of an autocorrelator and an optical spectrum analyzer. We find that the pump pulses have a chirped Gaussian profile with an unchirped pulse duration of 200 fs (FWHM) and a quadratic spectral phase profile $b \omega^{2}$, with $b=4.3 \times$ $10^{-27} \mathrm{~s}^{2}$, where $\omega$ is the angular frequency difference from the carrier pump frequency. The signal pulses have a chirped hyperbolic secant profile of 152-fs (FWHM) unchirped pulse duration and a chirp characterized by $b=$ $2.3 \times 10^{-27} \mathrm{~s}^{2}$.

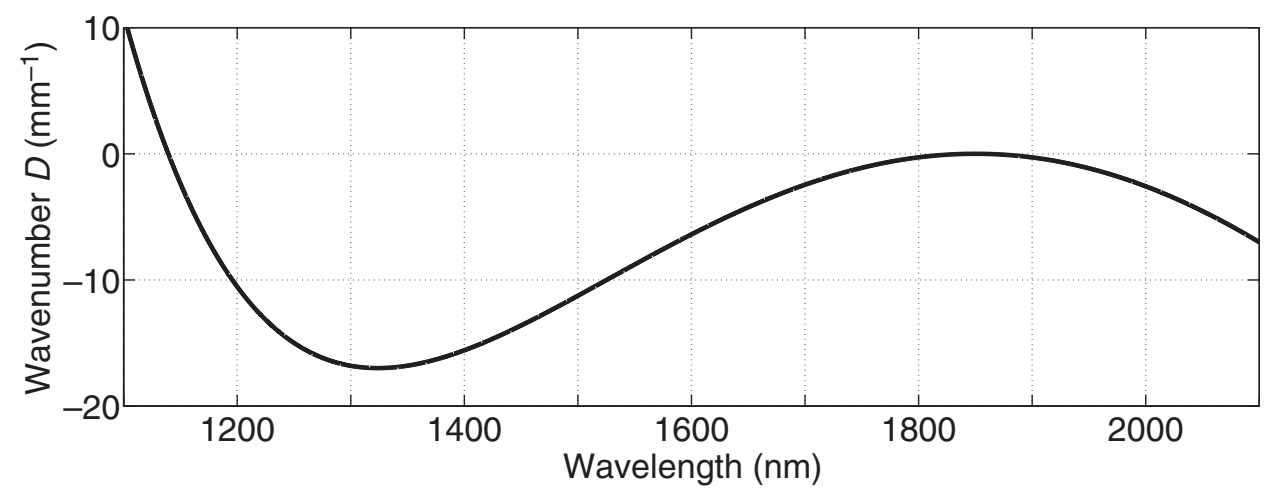

FIG. 1. Wavenumber $D$ of the quasi-TE fundamental mode as a function of the wavelength for a $220 \times 789 \mathrm{~nm}^{2}$ silicon waveguide. See Eq. (3) for the definition of $D$. The group velocity, in the reference frame moving at the group velocity of the pump at $1850 \mathrm{~nm}$, is proportional to the slope of the curve. 

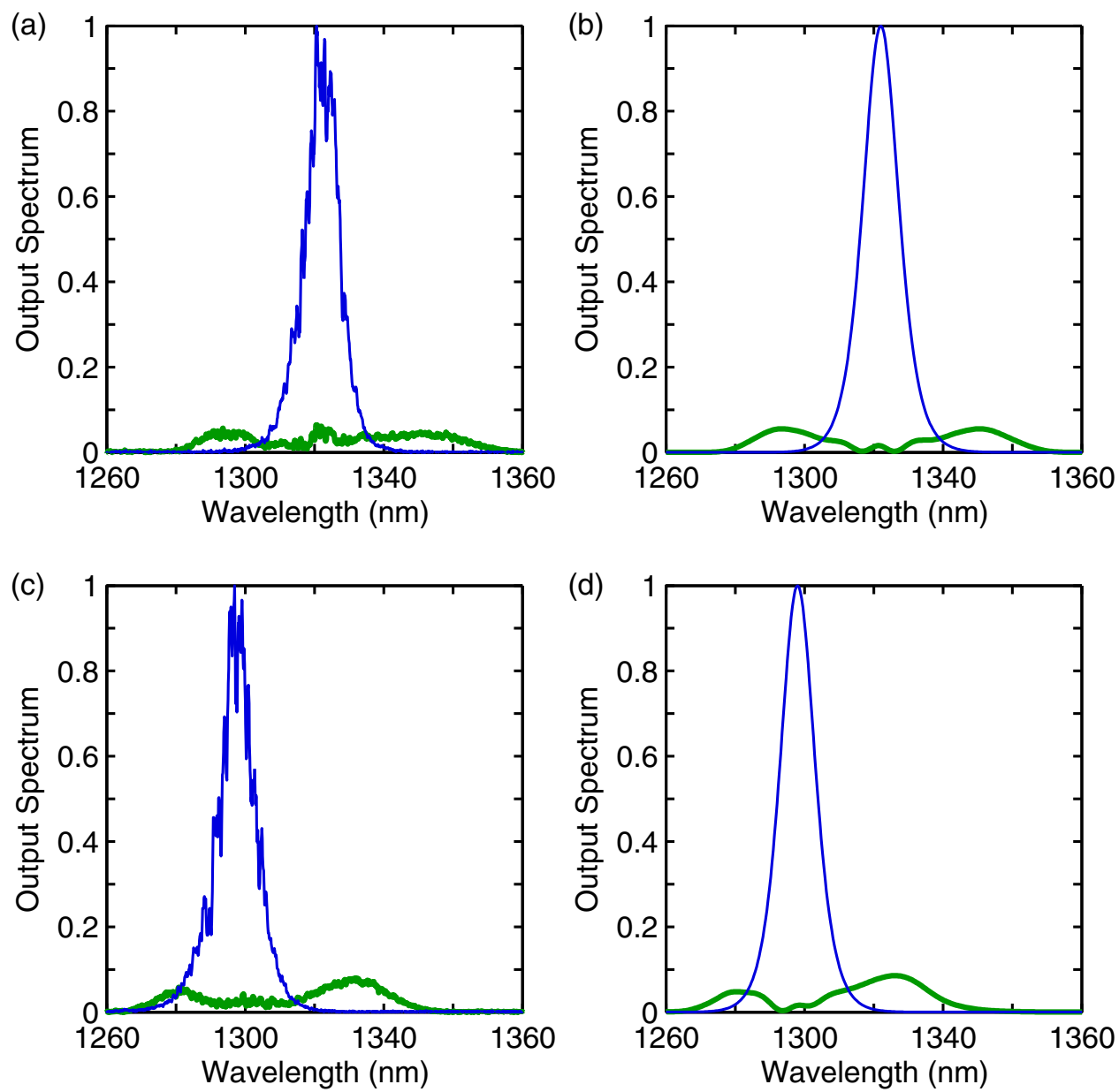

FIG. 3. Output signal spectrum without (thin blue line) and with (thick green line) the pump pulse. Experiments at the GVM wavelength of $1322 \mathrm{~nm}$ for the signal (a) and at $1298 \mathrm{~nm}$ (c); (b),(d) corresponding simulations by means of the CGNLSE. The spectra are normalized to the output-signal peak spectral density without the pump. The simulation parameters are as follows: pump peak power $P_{p}=3.5 \mathrm{~W}(61-\mu \mathrm{W}$ average input) and delay 0 fs (b); and $P_{p}=2.5 \mathrm{~W}(43-\mu \mathrm{W}$ average input) and delay $-100 \mathrm{fs}(\mathrm{d})$. The collected output average power is measured to be $0.8 \mu \mathrm{W}$ (a) and 0.5 $\mu \mathrm{W}$ (c). The difference between the input power in the simulation and the experimental output power comes from the nonlinear propagation loss and the output coupling loss.
From the waveguide-dispersion curve, we can see that the wavelength of $1322 \mathrm{~nm}$ is group velocity matched (GVM) with the pump at $1848 \mathrm{~nm}$. For slightly detuned signals and proper initial delays, the cross-phase modulation between the pump and the signal waves leads to a partial reflection of the signal pulse in the time reference frame of the pump. Simultaneously, the reflected pulse experiences a frequency shift across the GVM frequency such that it preserves the wavenumber $D$ [22]. The signal pulse could, in principle, be totally reflected by the pump pulse, but we expect the ND TPA to reduce the efficiency of the process.

The output spectra with and without the pump are shown in Figs. 3(a) and 3(c). We can readily notice that the interaction with the pump pulse dramatically decreases the output signal power, showing that the cross-TPA is quite large. Moreover, the shape of the signal spectrum is distorted because of the cross-phase modulation with the pump. For a signal at the GVM wavelength [see Fig. 3(a)] and a zero delay between the two input pulses, the shape of the spectrum stays symmetric around the signal carrier frequency. Conversely, in the case of a delayed input signal at $1298 \mathrm{~nm}$, the spectrum does not remain symmetric because of the partial reflection of the signal pulse on the pump pulse [see Fig. 3(c)]. The initial delay between the pump and the signal is experimentally set to maximize the frequency peak around $1330 \mathrm{~nm}$.

Next we study the efficiency of the cross-absorption as a function of the initial delay between the pump and the signal. The cross-TPA is an extremely fast nonlinear effect, in contrast to the absorption mediated by photoinduced free carriers generated by self-TPA or cross-TPA. We thus expect the signal transmission to be highly sensitive to the pump-signal delay. Figures 4(a) and 4(b) show the transmitted signal energy, normalized with respect to the maximum transmission, as a function of this delay. For the GVM case [Fig. 4(a)], two different pump powers are considered. The results show a fast response time $(\ll 100 \mathrm{fs})$, confirming the electronic origin of the nonlinear absorption. Moreover, as there are no significant transmission differences between large positive and large negative delays, the free carriers do not play a role in the experiment, in contrast to what was found in Refs. [15,16]. The curves cannot be considered as cross-correlation traces between the pump and the signal because both pulses are distorted when propagating in the dispersive nonlinear waveguide. 


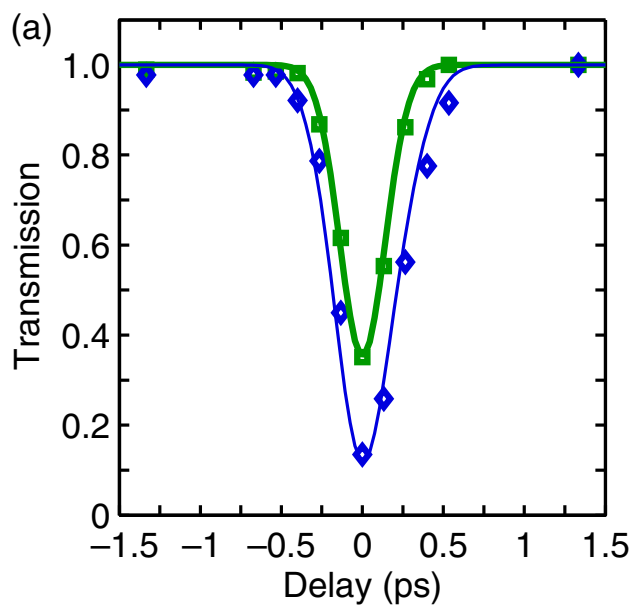

\section{MODELING}

\section{A. Coupled-wave model}

To compare our experimental results with theory, we first consider the coupled-equation model commonly used to describe the interaction between the two copropagating pulses [20]. These equations are derived assuming that the cross-TPA coefficient $\beta_{\mathrm{TPA}}\left(\omega_{1}, \omega_{2}\right)$ and the crossphase-modulation coefficient $n_{2}\left(\omega_{1}, \omega_{2}\right)$ can be replaced by their degenerate value at the mean frequency. Studies have demonstrated that the Raman effect is negligible when one is dealing with short pulses in silicon $[5,23]$, so we do not take it into account. The coupled nonlinear Schrödinger equations thus read

$$
\begin{aligned}
& \frac{\partial E_{p}(z, t)}{\partial z}-\mathcal{F}^{-1}\left[i D_{p}(\omega) \tilde{E}_{p}(z, \omega)\right]+\frac{1}{2}\left(\alpha_{p}+\sigma N_{c}\right) E_{p} \\
& \quad+i k_{0 p} k_{c} N_{c} E_{p}-i\left(\gamma_{p p}\left|E_{p}\right|^{2}+2 \gamma_{p s}\left|E_{s}\right|^{2}\right) E_{p}=0, \\
& \frac{\partial E_{s}(z, t)}{\partial z}-\mathcal{F}^{-1}\left[i D_{s}(\omega) \tilde{E}_{s}(z, \omega)\right]+\frac{1}{2}\left(\alpha_{s}+\sigma N_{c}\right) E_{s} \\
& \quad+i k_{0 s} k_{c} N_{c} E_{s}-i\left(\gamma_{s s}\left|E_{s}\right|^{2}+2 \gamma_{s p}\left|E_{p}\right|^{2}\right) E_{s}=0,
\end{aligned}
$$

where $E_{p}(z, t)$ and $E_{s}(z, t)$ describe the slowly varying envelope of the pump and signal fields as a function of the propagation distance $z$ and time $t$, and $\tilde{E}(z, \omega)=\mathcal{F}[E(z, t)]$ is the Fourier transform of the field $\left(\mathcal{F}^{-1}\right.$ denotes the inverse Fourier transform).

The dispersion operator at the pump frequency is defined as

$$
D_{p}(\omega)=\beta(\omega)-\beta\left(\omega_{p}\right)-\left.[\partial \beta / \partial \omega]\right|_{\omega_{p}}\left(\omega-\omega_{p}\right),
$$

where $\beta(\omega)$ is the frequency-dependent wavenumber and $\omega_{p}$ denotes the pump frequency. At the signal frequency $\omega_{s}$, the dispersion operator is $D_{s}(\omega)=$ $D_{p}\left[\omega-\left(\omega_{s}-\omega_{p}\right)\right]$. The wavenumber $\beta(\omega)$ is numerically
FIG. 4. Normalized transmission of the signal as a function of the the input signal-pump delay for a pump wavelength of 1848 nm. (a) Experiments at the GVM signal wavelength (1322 nm) for an average collected power of $1.6 \mu \mathrm{W}$ (diamonds) and $0.3 \mu \mathrm{W}$ (squares). Simulations for $P_{p}=6$ $\mathrm{W}$ (thin blue line; average input power $104 \mu \mathrm{W}$ ) and $1.5 \mathrm{~W}$ (thick green line; average input power $26 \mu \mathrm{W}$ ). (b) Experiments at 1298 $\mathrm{nm}$ for an average collected power of $0.25 \mu \mathrm{W}$ (squares) and simulation for $P_{p}=1 \mathrm{~W}$ (average power $17 \mu \mathrm{W})$.

computed by use of a mode solver (Lumerical). $\alpha_{p}$ and $\alpha_{s}$ characterize the linear loss experienced by the pump and the signal. These two coefficients where experimentally evaluated at $2 \mathrm{~dB} / \mathrm{cm}$.

The effect of photogenerated free carriers of density $N_{c}$ is taken into account. $\sigma N_{c}$ corresponds to the free-carrierinduced loss, and the carrier density $N_{c}$ is calculated by solving the rate equation:

$$
\begin{aligned}
\frac{\partial N_{c}(z, t)}{\partial t}= & \frac{\operatorname{Im}\left[\gamma_{p p}\right]}{\hbar \omega_{p} A_{p p}}\left|E_{p}(z, t)\right|^{4}+\frac{\operatorname{Im}\left[\gamma_{s s}\right]}{\hbar \omega_{s} A_{p p}}\left|E_{s}(z, t)\right|^{4} \\
& +4 \frac{\operatorname{Im}\left[\gamma_{p s}\right]}{\hbar \omega_{p} A_{p s}}\left|E_{p}(z, t)\right|^{2}\left|E_{s}(z, t)\right|^{2}-\frac{N_{c}(z, t)}{\tau_{c}} .
\end{aligned}
$$

The complex nonlinear parameters $\gamma_{\mu \nu}$ account for the self- and cross- amplitude and phase modulations. They are given by

$$
\gamma_{\mu \nu}=\frac{\omega_{\mu} \eta_{\mu \nu} \bar{n}^{2} n_{2}(\bar{\omega})}{c \sqrt{A_{\mu \mu} A_{\nu \nu}} n_{\mu} n_{\nu}}+i \frac{\omega_{\mu} \eta_{\mu \nu} \bar{n}^{2} \beta_{\mathrm{TPA}}(\bar{\omega})}{2 \bar{\omega} \sqrt{A_{\mu \mu} A_{\nu \nu}} n_{\mu} n_{\nu}}
$$

where $A_{v v}$ and $n_{v}$ are the effective area related to a thirdorder nonlinear process and the mode index, at the frequency $\omega_{\nu}$, calculated with the mode solver; $\bar{n}$ is the mode index at the average frequency $\bar{\omega}=\left(\omega_{\mu}+\omega_{\nu}\right) / 2 ; n_{2}$ and $\beta_{\text {TPA }}$ are the Kerr nonlinear refractive index and the TPA coefficient; and $\eta_{\mu \nu}$ are the mode-overlap factors as defined in Ref. [20]. The computed effective areas are $A_{p p}=$ $0.241 \mu \mathrm{m}^{2}, A_{s s}=0.147 \mu \mathrm{m}^{2}$, and $A_{p s}=0.176 \mu \mathrm{m}^{2}$, and the complex nonlinear coefficients are, on the basis of the data from Ref. [13],

$$
\begin{aligned}
& \gamma_{p p}=(164+14 i) \mathrm{W}^{-1} \mathrm{~m}^{-1}, \\
& \gamma_{s s}=(154+59 i) \mathrm{W}^{-1} \mathrm{~m}^{-1}, \\
& \gamma_{p s}=(97+31 i) \mathrm{W}^{-1} \mathrm{~m}^{-1}, \\
& \gamma_{s p}=(138+45 i) \mathrm{W}^{-1} \mathrm{~m}^{-1} .
\end{aligned}
$$


We can see that the real part of $\gamma$ is almost identical for the pump and the signal. Conversely, the self-TPA coefficient varies by more than a factor of 4 (see also Fig. 5), and the signal cross-TPA coefficient is three times larger than the self-TPA coefficient at the pump wavelength.

The coupled generalized nonlinear Schrödinger equations (CGNLSEs) [Eqs. (1) and (2)] are appropriate for modeling the interaction of the two waves since their spectra do not overlap. This set of equations is numerically integrated by a split-step Fourier method, with the input pump power as the only free parameter (all the other parameters are either computed or precisely measured). Remarkably, all the experimental results can be very well reproduced by numerical simulations. The amplitude and the shape of the transmitted signal agree when both the signal and the pump propagate with the same group velocity [Figs. 3(a) and 3(b)] and when the signal is slightly slower than the pump pulse [Figs. 3(c) and 3(d)]. There is also very good agreement for the transmission of the signal energy as a function of the relative delay between the signal and the pump (see Fig. 4). These results confirm that a $10-\mathrm{dB}$ ultrafast attenuation of the signal can be obtained with a pump pulse energy as low as $1.2 \mathrm{pJ}$ (6-W peak power). This energy is lower than the energies reported at telecom wavelengths for quasidegenerate TPA in silicon [15] and in amorphous silicon [24] because of the large cross-absorption of the signal but a low TPA at the pump wavelength. This shows the potential of silicon for
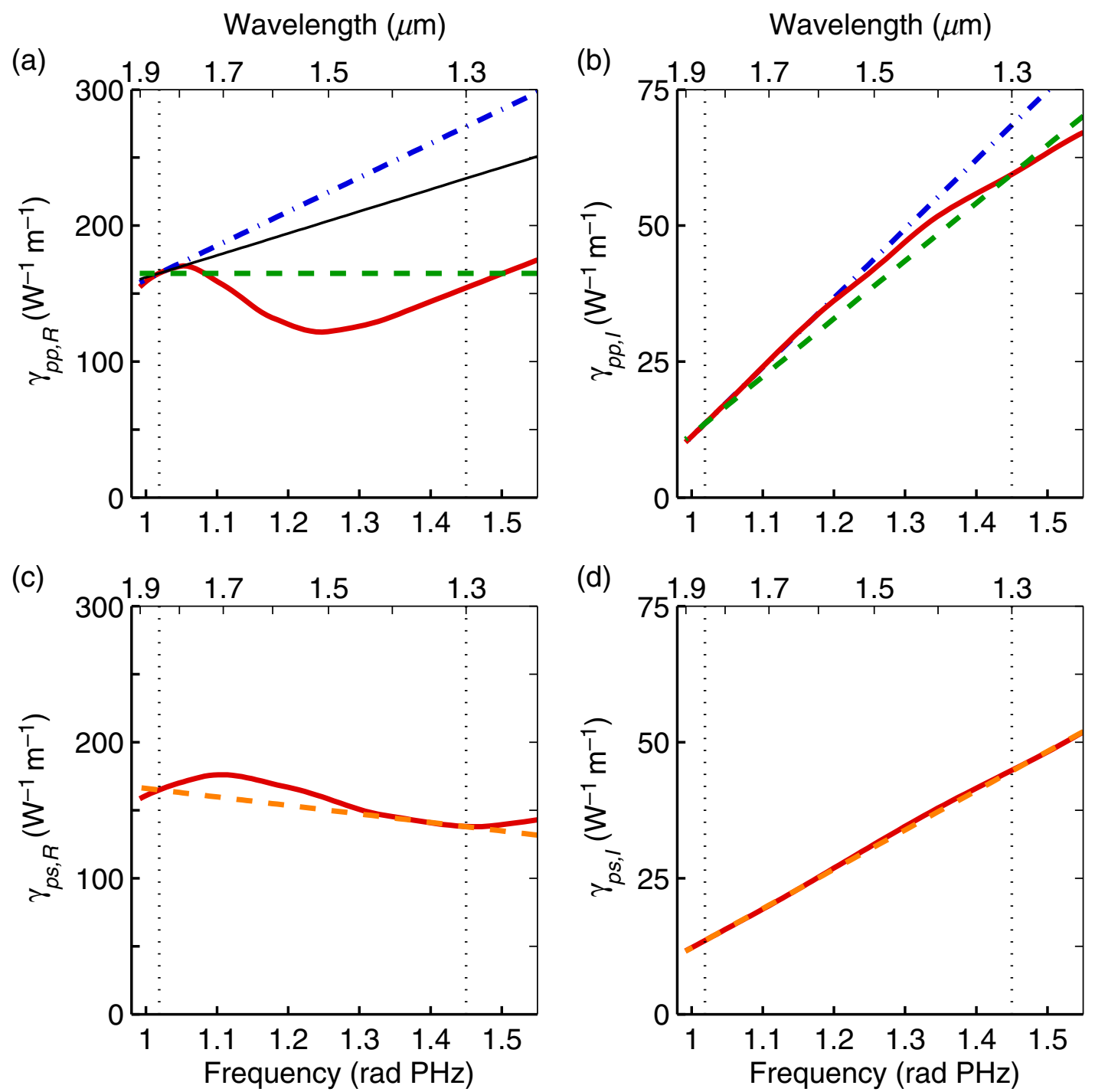

FIG. 5. Dispersion of the degenerate real (a) and imaginary (b) parts of the nonlinear coefficient $\gamma$ (thick red curve). The vertical dotted lines correspond to the angular frequency of the pump (1.02 rad PHz) and the signal (1.45 rad PHz). The thin black line in (a) shows the dispersion corresponding to $s_{R}=1 / \omega_{p}$. The dash-dotted blue lines show the first-order linear approximation, and the dashed green lines correspond to $s_{R}=0 \mathrm{fs}$ and $s_{I}=7.5 \mathrm{fs}$. Dispersion of the real (c) and imaginary (d) parts of the cross nonlinear coefficient $\gamma_{p s}$ (red curve). The dashed orange lines correspond to $s_{R}=-0.39 \mathrm{fs}$ and $s_{I}=5.15 \mathrm{fs}$. See also Fig. 6. 
ultrafast all-optical signal processing with spectrally distant waves. These results also demonstrate that owing to the short pulse duration, the density of photogenerated carriers is too low to significantly impact the cross-absorption dynamics, even if the energy of the pump photons is still above the half-band-gap. Simulations for large delays between the pump and the signal reveal that the free-carrier absorption is responsible for only $2 \%$ of the signal attenuation. Finally, the excellent agreement between theory and experiment confirms that the simplification of reducing the nondegenerate nonlinear coefficients $n_{2}\left(\omega_{p}, \omega_{s}\right)$ and $\beta_{\mathrm{TPA}}\left(\omega_{p}, \omega_{s}\right)$ to their value at the average frequency $\bar{\omega}$ is valid for indirect-band-gap semiconductors, at least for photon energy in the range from $0.6 E_{g}$ to $0.85 E_{g}$.

\section{B. Single equation}

The nonlinear propagation of broadband waves in integrated nanophotonic wire waveguides has recently attracted a lot of attention for its potential for efficient generation of a supercontinuum or frequency comb at low power, or for on-chip generation of ultrashort pulses through temporal pulse compression of high-order solitons. To describe the propagation of these waves in nanophotonic waveguides, the generalized nonlinear Schrödinger equation (GNLSE) is widely used. It has historically been successfully applied to describe the nonlinear pulse propagation in optical fibers. In such media, for moderate pulse peak power, nonlinear absorption is very low and thus negligible. The effect of the dispersion of the nonlinear parameters was first discussed in the context of pulse propagation in optical fibers and applied to SCG [25-27]. In these studies, a linear dispersion of $\gamma_{R}$, known as the "self-steepening term," was considered in the GNLSE. It was later included in models for the simulation of SCG in integrated waveguides made up of various nonlinear media such as chalcogenide glasses [28], silicon [5,20,29,30], amorphous silicon [31], silicon nitride [32], or indium gallium phosphide [33], where qualitatively good agreements between the simulations and the experiments have been reported. Higher-order dispersion terms were considered in Ref. [34]. Apart from this later work, the dispersion of the nonlinear absorption was not specifically taken into account. However, the dispersion of the TPA is not negligible and may have a larger impact on the nonlinear interaction than the dispersion of the Kerr effect.

The dispersion of the real $\left(\gamma_{R}\right)$ and the imaginary $\left(\gamma_{I}\right)$ parts of the nonlinear coefficient $\gamma$ for self-induced nonlinear refraction and absorption is plotted in Fig. 5 (see the red lines). For the silicon waveguide under consideration, the dispersion of the TPA coefficient is almost linear. Its relative variation is larger than that for the nonlinear refraction coefficient, which tends to oscillate around a constant value of about $150 \mathrm{~W}^{-1} \mathrm{~m}^{-1}$ in the wavelength range of interest $(1.2-1.9 \mu \mathrm{m})$. Also plotted in Figs. 5(a) and 5(b) are the first-order approximations of the dispersion of the nonlinear parameters (dash-dotted blue lines). For the real part of $\gamma$, we thus have in the spectral domain $\gamma_{R}(\omega)=\gamma_{R}\left(\omega_{p}\right) \times$ $\left[1+s_{R}\left(\omega-\omega_{p}\right)\right]$, with $s_{R}=\gamma_{R}^{-1}\left[\partial \gamma_{R} / \partial \omega\right]_{\omega_{p}}$. Sometimes the dispersion of $n_{2}, n_{\mu \mu}$, and the effective area $A_{\mu \mu}$ is ignored [31], leading to a value of $s_{R}=1 / \omega_{p}$ (thin black line). We can see that this approximation strongly deviates from $\gamma_{R}(\omega)$ and clearly overestimates the self-modulation and the cross-phase modulation at frequencies higher than that of the pump. A constant $\gamma_{R}$ approximation would be more accurate (dashed green line). On the other hand, not taking the dispersion of the TPA into account leads to an underestimation of the cross-TPA.

We separately consider the dispersion of the TPA and of the Kerr effect in the GNLSE, which thus reads

$$
\begin{aligned}
& \frac{\partial E(z, t)}{\partial z}-\mathcal{F}^{-1}[i D(\omega) \tilde{E}(z, \omega)] \\
& \quad+\frac{\alpha}{2} E-i\left\{\left[\gamma_{R}\left(1+i s_{R} \frac{\partial}{\partial t}\right)\right]\right. \\
& \left.\quad+i\left[\gamma_{I}\left(1+i s_{I} \frac{\partial}{\partial t}\right)\right]\right\}|E|^{2} E=0,
\end{aligned}
$$

where the free-carrier effects are ignored and where $s_{R}$ and $s_{I}$ are the characteristic times associated with the linear dispersion of the nonlinear coefficients $\gamma_{R}$ and $\gamma_{I}$.

Equation (6) is not strictly equivalent to the coupled equations [Eqs. (1) and (2)] [35]. The GNLSE can indeed not properly account for both the self-effects and the cross-effects since it implies that $\gamma_{p s}=\gamma_{p p}$ and $\gamma_{s p}=\gamma_{s s}$, which is not verified for spectrally distant waves (see, e.g., Fig. 5).

Figure 6 shows the simulations of the interaction between the signal and the pump with different characteristic times $s_{R}$ and $s_{I}$ in Eq. (6). These results are compared with the numerical integration of Eqs. (1) and (2) considered as a benchmark model for modeling interactions between the two waves. Firstly, we consider the GNLSE without taking the dispersion of the TPA and Kerr coefficients into account $\left[\gamma_{R}=\operatorname{Re}\left(\gamma_{p p}\right)\right.$ and $\left.\gamma_{I}=\operatorname{Im}\left(\gamma_{p p}\right)\right]$. The cross-TPA experienced by the signal pulse is less than its actual value. The output spectral density is clearly overestimated compared with that from the experiment and the CGNLSEs. A first-order approximation of the dispersion of the real and the imaginary parts of $\gamma$ already improves the result. However, the cross-TPA and the cross-phase modulation are now overestimated, as seen in Fig. 5. A better agreement with the CGNLSEs is obtained when the dispersion of $\gamma_{R}$ is neglected $\left(s_{R}=0\right)$ and $s_{I}$ is computed so that $\gamma_{I}$ takes its correct value both at the pump wavelength and at the signal wavelength; that is, $s_{I}=1 / \gamma_{I}\left(\omega_{p}\right) \times\left[\gamma_{I}\left(\omega_{p}\right)-\gamma_{I}\left(\omega_{s}\right)\right] /\left[\omega_{p}-\omega_{s}\right]=7.5 \mathrm{fs}$ (see also the green curves in Fig. 5). Nevertheless, there is still a discrepancy between the CGNLSEs and the GNLSE 


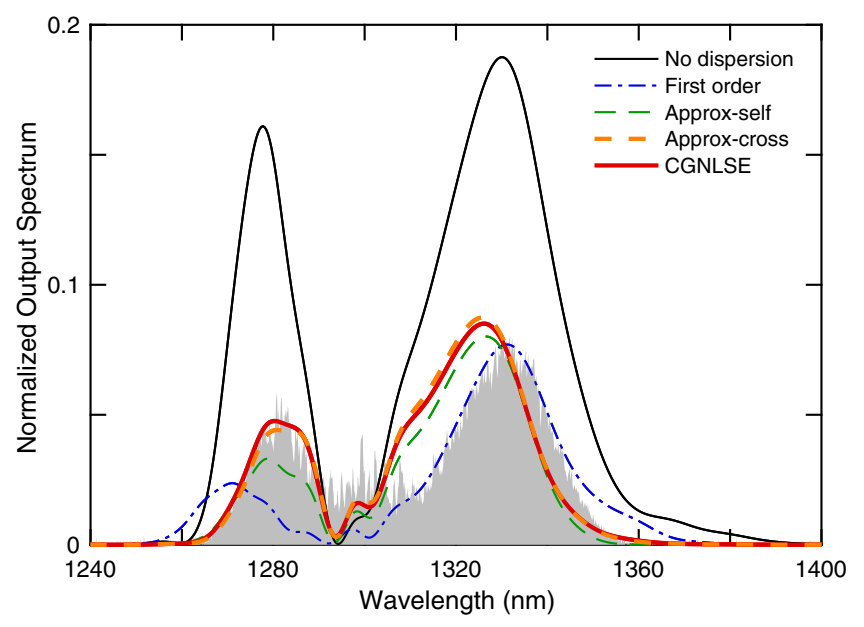

FIG. 6. Comparison between the different models. No dispersion, GNLSE without dispersion of the nonlinearity $\left(s_{R}=0\right.$ and $s_{I}=0$ ); first order, GNLSE with characteristic times corresponding to the first-order approximation of the nonlinear dispersion $\left(s_{R}=1.5\right.$ fs and $s_{I}=9 \mathrm{fs}$, see also the blue lines in Fig. 5); approx-self, GNLSE with $s_{R}=0$ fs and $s_{I}=7.5 \mathrm{fs}$, corresponding to the dashed green lines in Fig. 5; approx-cross, GNLSE so that the cross nonlinear coefficients at the signal agree with Eqs. (1) and (2) $\left(s_{R}=-0.39\right.$ fs and $\left.s_{I}=5.15 \mathrm{fs}\right)$; CGNLSE, simulation with the CGNLSEs [Eqs. (1) and (2)]. The pump is at $1848 \mathrm{~nm}$ with $P_{p}=2.5 \mathrm{~W}$, and the input signal wavelength is $\lambda_{s}=1298 \mathrm{~nm}$. The normalization is the same as in Fig. 3. The shaded gray area shows the signal output experimental spectrum.

because the cross-absorption coefficient considered is 59 $\mathrm{W}^{-1} \mathrm{~m}^{-1}$ instead of $45 \mathrm{~W}^{-1} \mathrm{~m}^{-1}$. As long as the crossinteractions with the pump dominate the nonlinear effects at the signal wavelength, the dispersion of the cross coefficient $\gamma_{s p}$ should be considered. The coefficients $s_{R}$ and $s_{I}$ can thus be set such that the cross-phase modulation and the cross-absorption at the signal wavelength take the same values as in Eq. (2). We can see in Figs. 5(c) and 5(d) that these linear approximations are actually very close to $\gamma_{p s}$ over the whole frequency range between the pump and the signal. The two models now give similar results. The small deviations between the two models likely come from the effects of the free carriers that are not taken into account in Eq. (6). For historical reasons, only the dispersion of the Kerr nonlinearity coefficient is usually considered for modeling the nonlinear propagation in integrated semiconductor waveguides. However, for silicon, it is now evident that the dispersion of the TPA coefficient should not be ignored, while a constant Kerr coefficient might give better results than a linear approximation around the pump frequency.

\section{CONCLUSION}

In this work we experimentally investigate the nonlinear interaction between two subpicosecond pulses through cross-TPA and cross-phase modulation in silicon wire waveguides. We consider an experiment in which a signal at about $1330 \mathrm{~nm}$ is interacting with a pump propagating in the anomalous dispersion at a wavelength $(1850 \mathrm{~nm})$ close to the semiconductor half-band-gap. The waveguide dispersion is engineered so that the pump and the signal propagate at almost the same group velocity. The spectrum of the signal at the output of the waveguide is recorded for different input signal wavelengths and different input pump delays. The experiment shows strong absorption of the signal due to a large cross-TPA with the pump. For subpicosecond pulses, the dynamics of the cross nonlinear absorption is dominated by the electronic ultrafast TPA. The free-carrier absorption is negligible as the TPA of the pump is low for wavelengths close to or beyond the half-band-gap. A 10-dB modulation of the transmitted signal is measured for a pump pulse energy of $1.2 \mathrm{pJ}$. The experimental results are compared with the numerical integration of a set of two CGNLSEs. Very good agreements are obtained for the pump-signaldelay-dependent transmission and for the output spectra. From these results, we conclude that, in indirect-band-gap semiconductors, the simplification of computing the cross nonlinear coefficients from the degenerate $n_{2}$ and $\beta_{\text {TPA }}$ at the average frequency is a valid approximation. This could be helpful for optimizing optical functions resorting to cross-interactions between two waves at very different wavelengths. The commonly used single GNLSE is also considered for simulation of the nonlinear interaction in the waveguide. The dispersion of the Kerr coefficient and of the nonlinear absorption is taken into account. It appears that the dispersion of the TPA cannot be ignored. Moreover, in this study the standard first-order approximation of the nonlinear effective Kerr coefficient is not appropriate, and a constant real part of $\gamma$ gives better results. The best results are, however, obtained when the coefficients $s_{R}$ and $s_{I}$ are such that they properly account for cross nonlinear effects. This observation might impact simulations and analytic studies of SCG and frequency-comb generation in integrated semiconductor structures that are based on the GNLSE model. A large variation of the nonlinear parameters and of the effective area over the spectrum is indeed encountered in these structures.

\section{ACKNOWLEDGMENTS}

This work was supported by the Belgian Federal Science Policy Office (BELSPO) Interuniversity Attraction Pole project Photonics@be and by the Fonds de la Recherche Fondamentale Collective (Grant No. PDR.T.1084.15).

[1] F. Li, M. Pelusi, D.-X. Xu, A. Densmore, R. Ma, S. Janz, and D. J. Moss, Error-free all-optical demultiplexing at 160 $\mathrm{gb} / \mathrm{s}$ via FWM in a silicon nanowire, Opt. Express 18, 3905 (2010). 
[2] R. Dekker, A. Driessen, T. Wahlbrink, C. Moormann, J. Niehusmann, and M. Först, Ultrafast Kerr-induced alloptical wavelength conversion in silicon waveguides using $1.55 \mu \mathrm{m}$ femtosecond pulses, Opt. Express 14, 8336 (2006).

[3] M. A. Foster, A. C. Turner, R. Salem, M. Lipson, and A. L. Gaeta, Broadband continuous-wave parametric wavelength conversion in silicon nanowaveguides, Opt. Express 15, 12949 (2007).

[4] I.-W. Hsieh, X. Chen, X. Liu, J. I. Dadap, N. C. Panoiu, C.-Y. Chou, F. Xia, W. M. Green, Y. A. Vlasov, and R. M. Osgood, Supercontinuum generation in silicon photonic wires, Opt. Express 15, 15242 (2007).

[5] F. Leo, S.-P. Gorza, J. Safioui, P. Kockaert, S. Coen, U. Dave, B. Kuyken, and G. Roelkens, Dispersive wave emission and supercontinuum generation in a silicon wire waveguide pumped around the $1550 \mathrm{~nm}$ telecommunication wavelength, Opt. Lett. 39, 3623 (2014).

[6] T. K. Liang, H. K. Tsang, I. E. Day, J. Drake, A. P. Knights, and M. Asghari, Silicon waveguide two-photon absorption detector at $1.5 \mu \mathrm{m}$ wavelength for autocorrelation measurements, Appl. Phys. Lett. 81, 1323 (2002).

[7] T. K. Liang, L. R. Nunes, T. Sakamoto, K. Sasagawa, T. Kawanishi, M. Tsuchiya, G. R. A. Priem, D. V. Thourhout, P. Dumon, R. Baets, and H. K. Tsang, Ultrafast all-optical switching by cross-absorption modulation in silicon wire waveguides, Opt. Express 13, 7298 (2005).

[8] D. Moss, L. Fu, I. Littler, and B. Eggleton, Ultrafast alloptical modulation via two-photon absorption in silicon-oninsulator waveguides, Electron. Lett. 41, 320 (2005).

[9] P. Mehta, N. Healy, T. D. Day, J. R. Sparks, P. J. A. Sazio, J. V. Badding, and A. C. Peacock, All-optical modulation using two-photon absorption in silicon core optical fibers, Opt. Express 19, 19078 (2011).

[10] E.-K. Tien, N. S. Yuksek, F. Qian, and O. Boyraz, Pulse compression and modelocking by using TPA in silicon waveguides, Opt. Express 15, 6500 (2007).

[11] Y. Yue, H. Huang, L. Zhang, J. Wang, J.-Y. Yang, O. F. Yilmaz, J. S. Levy, M. Lipson, and A. E. Willner, UWB monocycle pulse generation using two-photon absorption in a silicon waveguide, Opt. Lett. 37, 551 (2012).

[12] C. Ciret, S.-P. Gorza, G. Roelkens, B. Kuyken, C. Husko, and F. Leo, arXiv:1805.05175.

[13] A. D. Bristow, N. Rotenberg, and H. M. van Driel, Twophoton absorption and Kerr coefficients of silicon for 850-2200 nm, Appl. Phys. Lett. 90, 191104 (2007).

[14] J. L. Cheng, J. Rioux, and J. E. Sipe, Full band structure calculation of two-photon indirect absorption in bulk silicon, Appl. Phys. Lett. 98, 131101 (2011).

[15] S. Suda, T. Ogasawara, Y. Shoji, K. Kintaka, H. Kawashima, T. Hasama, and H. Ishikawa, Femtosecond Index Dynamics in Silicon Wire Waveguides, in 6th IEEE International Conference on Group IV Photonics (IEEE, 2009), p. 98-100.

[16] Y. Zhang, C. Husko, S. Lefrancois, I. H. Rey, T. F. Krauss, J. Schröder, and B. J. Eggleton, Non-degenerate two-photon absorption in silicon waveguides: Analytical and experimental study, Opt. Express 23, 17101 (2015).

[17] R. Sarkissian and J. O'Brien, Cross two photon absorption in a silicon photonic crystal waveguide fiber taper coupler with a physical junction, J. Appl. Phys. 117, 033101 (2015).

[18] C. M. Cirloganu, L. A. Padilha, D. A. Fishman, S. Webster, D. J. Hagan, and E. W. V. Stryland, Extremely nondegenerate two-photon absorption in direct-gap semiconductors [Invited], Opt. Express 19, 22951 (2011).

[19] D. A. Fishman, C. M. Cirloganu, S. Webster, L. A. Padilha, M. Monroe, D. J. Hagan, and E. W. V. Stryland, Sensitive mid-infrared detection in wide-bandgap semiconductors using extreme non-degenerate two-photon absorption, Nat. Photonics 5, 561 (2011).

[20] Q. Lin, O. J. Painter, and G. P. Agrawal, Nonlinear optical phenomena in silicon waveguides: Modeling and applications, Opt. Express 15, 16604 (2007).

[21] C. Ciret, F. Leo, B. Kuyken, G. Roelkens, and S.-P. Gorza, Observation of an optical event horizon in a silicon-oninsulator photonic wire waveguide, Opt. Express 24, 114 (2016).

[22] T. G. Philbin, C. Kuklewicz, S. Robertson, S. Hill, F. Konig, and U. Leonhardt, Fiber-optical analog of the event horizon, Science 319, 1367 (2008).

[23] L. Yin, Q. Lin, and G. P. Agrawal, Soliton fission and supercontinuum generation in silicon waveguides, Opt. Lett. 32, 391 (2007).

[24] Y. Shoji, T. Ogasawara, T. Kamei, Y. Sakakibara, S. Suda, K. Kintaka, H. Kawashima, M. Okano, T. Hasama, H. Ishikawa, and M. Mori, Ultrafast nonlinear effects in hydrogenated amorphous silicon wire waveguide, Opt. Express 18, 5668 (2010).

[25] K. Blow and D. Wood, Theoretical description of transient stimulated Raman scattering in optical fibers, IEEE J. Quantum Electron. 25, 2665 (1989).

[26] N. Karasawa, S. Nakamura, N. Nakagawa, M. Shibata, R. Morita, H. Shigekawa, and M. Yamashita, Comparison between theory and experiment of nonlinear propagation for a-few-cycle and ultrabroadband optical pulses in a fused-silica fiber, IEEE J. Quantum Electron. 37, 398 (2001).

[27] J. M. Dudley, G. Genty, and S. Coen, Supercontinuum generation in photonic crystal fiber, Rev. Mod. Phys. 78, 1135 (2006).

[28] M. R. E. Lamont, B. Luther-Davies, D.-Y. Choi, S. Madden, and B. J. Eggleton, Supercontinuum generation in dispersion engineered highly nonlinear $(\gamma=10 / \mathrm{W} / \mathrm{m}) \mathrm{As}_{2} \mathrm{~S}_{3}$ chalcogenide planar waveguide, Opt. Express 16, 14938 (2008).

[29] N. Singh, D. D. Hudson, Y. Yu, C. Grillet, S. D. Jackson, A. Casas-Bedoya, A. Read, P. Atanackovic, S. G. Duvall, S. Palomba, B. Luther-Davies, S. Madden, D. J. Moss, and B. J. Eggleton, Midinfrared supercontinuum generation from 2 to $6 \mu \mathrm{m}$ in a silicon nanowire, Optica 2, 797 (2015).

[30] A. Ishizawa, T. Goto, R. Kou, T. Tsuchizawa, N. Matsuda, K. Hitachi, T. Nishikawa, K. Yamada, T. Sogawa, and $\mathrm{H}$. Gotoh, Octave-spanning supercontinuum generation at telecommunications wavelengths in a precisely dispersion- and length-controlled silicon-wire waveguide with a double taper structure, Appl. Phys. Lett. 111, 021105 (2017).

[31] F. Leo, J. Safioui, B. Kuyken, G. Roelkens, and S.-P. Gorza, Generation of coherent supercontinuum in a-Si:H 
waveguides: Experiment and modeling based on measured dispersion profile, Opt. Express 22, 28997 (2014).

[32] A. R. Johnson, A. S. Mayer, A. Klenner, K. Luke, E. S. Lamb, M. R. E. Lamont, C. Joshi, Y. Okawachi, F. W. Wise, M. Lipson, U. Keller, and A. L. Gaeta, Octavespanning coherent supercontinuum generation in a silicon nitride waveguide, Opt. Lett. 40, 5117 (2015).

[33] U. D. Dave, C. Ciret, S.-P. Gorza, S. Combrie, A. D. Rossi, F. Raineri, G. Roelkens, and B. Kuyken, Dispersivewave-based octave-spanning supercontinuum generation in
In-GaP membrane waveguides on a silicon substrate, Opt. Lett. 40, 3584 (2015).

[34] L. Zhang, A. M. Agarwal, L. C. Kimerling, and J. Michel, Nonlinear Group IV photonics based on silicon and germanium: From near-infrared to mid-infrared, Nanophotonics 3, 247 (2014).

[35] See Supplemental Material at http://link.aps.org/supple mental/10.1103/PhysRevApplied.10.024033 for the relation between the CGNLSEs [Eqs. (1) and (2)] and the GNLSE (Eq. (6)). 